\title{
El uso de redes sociales y su implicación para la comunicación en salud. Revisión bibliográfica sobre el uso de Twitter y la enfermedad del cáncer
}

\author{
The use of social networks and their implication for health communication. A \\ bibliographic review on the use of Twitter and cancer
}

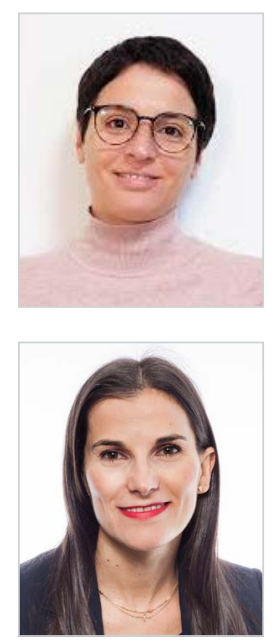

Belén Cambronero Saiz. Licenciada en Publicidad y Relaciones Públicas (UA) y Doctora en Estudios Pluridisciplinares de Género (líneas de Comunicación y Salud) (UA). Ha trabajado como investigadora FPI en el departamento de Comunicación y Psicología Social de la UA. En esta misma universidad ha participado en numerosos proyectos I+D+i y grupos de investigación, publicando más de una decena de artículos centrados en salud, comunicación y género. Desde 2016, trabaja como PDI en UNIR impartiendo docencia en Grado y Posgrado. Universidad Internacional de la Rioja, España

belen.cambronero@unir.net

ORCID: 0000-0001-5273-9534

Begoña Gómez Nieto. Licenciada en Publicidad y RR.PP. por la Universidad Complutense de Madrid y Doctora en Cultura y Comunicación por IE University. Acreditada como Profesora contratada doctora, ayudante doctora y profesora de universidad privada por ANECA y ACSUCYL. Coordinadora Académica del Máster en Comunicación e Identidad Corporativa de UNIR. Ha publicado más de setenta artículos en revistas de alto impacto y cuatro libros completos. Principales líneas de investigación: comunicación publicitaria, organizacional y periodística, ambient marketing, estructura de contenidos en webs, diferenciación de contenidos, igualdad de género en comunicación.

Universidad Internacional de la Rioja, España

begona.gomez.nieto@unir.net

ORCID: 0000-0002-1055-1864

Recibido: 30/05/2021 - Aceptado: 05/10/2021 - En edición: 21/10/2021 - Publicado: 01/12/2021

\section{Resumen:}

El cáncer es una de las enfermedades que más circula en las redes sociales debido a su alta incidencia, siendo Twitter una de las más efectivas para difundir información sobre este tema. Esta revisión tiene por objetivo compilar los principales hallazgos de los artículos que analizan la comunicación sobre cáncer en Twitter. Para ello se examinan las bases de datos de WoS y PubMed (2009-2019) y se realiza un análisis de contenido de los 20 artículos encontrados. El 64,3\% de los artículos concluyen afirmando que Twitter es una herramienta efectiva para
Received: 30/05/2021 - Accepted: 05/10/2021 - Early access: 21/10/2021 - Published: 01/12/2021

\section{Abstract:}

Cancer is one of the diseases that most circulates on social networks due to its high incidence. Twitter is one of the most effective networks for disseminating information on this topic. This review aims to compile the main findings of articles that analyse cancer communication on Twitter. For this purpose, we examined WoS and PubMed databases (2009-2019), and we carried out a content analysis of 20 articles found. $64.3 \%$ of the articles conclude that Twitter is an effective tool for health education and $58.3 \%$ consider that more significant

Cómo citar este artículo:

Cambronero Saiz, B. y Gómez Nieto, B. (2021). El uso de redes sociales y su implicación para la comunicación en salud. Revisión bibliográfica sobre el uso de Twitter y la enfermedad del cáncer. Doxa Comunicación, 33, pp. 377-392.

https://doi.org/10.31921/doxacom.n33a868 
educar en salud y un 58,3\% considera que todavía es necesaria una mayor intervención por parte de los profesionales sanitarios. Es por tanto una herramienta complementaria útil para la sensibilización y prevención de enfermedades, que todavía debe ir de la mano de otras acciones educativas y de alfabetización mediática.

\section{Palabras clave:}

Redes sociales; Twitter; cáncer; comunicación en salud; análisis bibliométrico. intervention by health professionals is still needed. Therefore, it is a useful complementary tool for disease awareness and prevention, which must still go hand in hand with other educational and media literacy actions.

\section{Keywords:}

Social network; Twitter; cancer; health communication; bibliometric analysis.

\section{Introducción}

Las redes sociales digitales como Twitter, Facebook e Instagram son un vehículo de comunicación esencial en la vida de los ciudadanos, llegando a sumar en la actualidad millones de usuarios en todo el mundo, con una constante tendencia creciente. En el año 2021, el número de usuarios asciende a 4.200 millones de personas, es decir, el 53,6\% de la población mundial, lo que supone un incremento usuarios activos respecto al 2020 del 13,2\% y un total de 490 millones de personas más (Galeano, 2021). Características como la agilidad, la inmediatez, la búsqueda de información o la posibilidad de generar y compartir contenidos son algunas de las razones que justifican el uso de estas redes, en las que de media se invierten más de dos horas diarias.

A pesar de que en el ranking de redes sociales digitales con mayor número de usuarios Twitter se posicione por debajo de, entre otras, Facebook (2,7 millones de usuarios), YouTube (2,2 millones de usuarios), Instagram (1,2 millones de usuarios) o TikTok (689 mil usuarios), esta red social se mantiene estable en el tiempo, con usuarios fieles que no la abandonan (We are social, 2021). Para Crua (2020), Twitter es la plataforma de microblogging por excelencia que se caracteriza por su instantaneidad y conexión en directo entre los usuarios, cuyo perfil es mayoritariamente masculino y de un rango de edad comprendido entre los 25 y 49 años.

Por otro lado, también hay que destacar que Twitter no sólo ha llegado a ser una plataforma para usuarios, sino que además en múltiples casos, se conforma como un canal habitual de trabajo para diversos profesionales. En Estados Unidos, se ha convertido en una práctica habitual para la policía y los cuerpos de bomberos de todo el país. Los primeros comunican arrestos, homicidios, revueltas, accidentes de tráfico, cortes en las carreteras, robos y avances en investigaciones criminales. En cuanto a los bomberos, lanzan mensajes para informar sobre sus salidas y reciben información de los ciudadanos, que les avisan, por ejemplo, de cambios en el viento en una zona incendiada que pueden ser claves para evitar que el fuego se propague (Sanz y Delgado, 2020).

Aunque sin duda, la característica fundamental de Twitter es su facilidad para que los usuarios puedan estar al día de nuevos eventos o noticias de actualidad (We are social, 2021). Algunos ejemplos de este tipo de uso los encontramos en la organización de manifestaciones o levantamientos sociales en Egipto e Irán (Castells, 2012), la retransmisión minuto a minuto del terremoto y posterior amenaza nuclear de Fukushima o durante el inicio de la pandemia del COVID-19, donde destacó como una herramienta muy poderosa para difundir información desde el ámbito de la salud pública. Los líderes mundiales y representantes de instituciones sanitarias de todo el mundo, la utilizaron para transmitir información a los ciudadanos de forma más rápida y directa que a través de los medios de comunicación tradicionales como la prensa, la radio o la televisión (Rufai y Bunce, 2020). 


\subsection{Alfabetización mediática y comunicación en salud a través de Twitter}

El uso de Twitter por las autoridades sanitarias se debe precisamente a que esta red social está posicionada como una de las más usadas para buscar y compartir información sobre salud (Matarin, 2015). Mediante el uso de hashtags miles de personas en diferentes lugares del mundo buscan información (Schapira, 2019) de carácter general, como hábitos de vida saludables o prevención de enfermedades, o de carácter específica, como la relacionada con la sintomatología de una enfermedad concreta o la profundización acerca de las implicaciones de un diagnóstico médico. Además, ha sido utilizado en investigación en salud para la vigilancia, educación y como una herramienta para la promoción, prevención y soporte al tratamiento de diversas condiciones, sin dejar de lado el potencial que representa para la promoción de la investigación, atendiendo al creciente número de publicaciones y la experiencia de diversos organismos que pretenden dar un impulso a la investigación utilizando este canal (Curioso y Carnero, 2011). Durante la pandemia de la COVID-19, usuarios de esta red se han configurado como auténticos portavoces con millones de seguidores, incluyendo medios de comunicación y funcionarios de gobierno (Sued y Cebral, 2020).

Además, gracias a la posibilidad de interacción entre los usuarios, Twitter puede ser una red de apoyo importante para los pacientes y sus familiares, al permitir que los implicados narren en ella sus experiencias personales y, al mismo tiempo, conozcan las opiniones y experiencias de otros en relación con un problema de salud, evitando así la denominada soledad del enfermo (Gage-Bouchard et al., 2018) y fomentando el asesoramiento entre usuarios (Keckley y Hoffmann, 2010). Es decir, mediante la participación pasan de ser meros consumidores de información a tener un papel mucho más activo y empoderado en materia de salud, convirtiéndose en "prosumidores" y generando información en base a su propio conocimiento (Martin y Tyner, 2012).

Sin embargo, esta nueva forma de generar conocimiento de manera participativa no está exenta de riesgos y conviene reflexionar sobre las implicaciones que pueden suponer la exposición a información sobre temas de salud con datos que en ocasiones pueden ser inciertos, estar sesgados o fundamentados en creencias, valores y prejuicios (Martín y Tyner, 2012).

De hecho, la infodemia relacionada con el COVID-19 evidenció que la pobre alfabetización en salud es un problema de salud pública subestimado a nivel mundial (Paakkari y Okan, 2020). Desde organizaciones internacionales como la UNESCO consideran que la alfabetización mediática e informacional es esencial para fortalecer la capacidad crítica de los ciudadanos frente a las fake news y la desinformación a través de bulos, muy frecuentes en las redes sociales (Calvo y Aruguete, 2020). Concretamente, esta alfabetización digital en el ámbito de la salud es esencial para empoderar a la población y evitar reforzar las diferencias sociales existentes y no generar nuevas desigualdades en base al dominio de la información sanitaria (Sanders et al., 2015).

En cuanto a los bulos, para Mateos, vicepresidente de la Asociación de Investigadores de la Salud (AIES) y coordinador de \#Salud sin bulos, señala en un estudio de EHON que éstos están causando mucho daño a los pacientes (García, 2018), ya que algunos dejan de tomar la medicación e incluso llegan a ocultar a su médico que están recurriendo a otros tratamientos complementarios, siendo necesario el contraste de la información con un profesional sanitario. Para González (2019), se presentan tres tipos de motivaciones a la hora de expandirlos: perjudicar a un tercero (los creadores de este bulo pretender sacar provecho del desprestigio de otros); ocultar el interés real de quien ha lanzado ese bulo (interés por vender productos); y/o crear alarma social.

Los profesionales del sector, conscientes de esta realidad, manifiestan la necesidad de intervenir utilizando los mismos canales para proporcionar al usuario información útil, y seguir desempeñando uno de sus roles más importantes: aportar información fiable al paciente y educar a la comunidad (Fernández et al., 2014). De esta manera, muchos sanitarios utilizan Twitter para pre- 
sentar estudios, opiniones, recomendaciones, directrices ante los distintos temas de salud contribuyendo a la alfabetización en salud de sus seguidores (Piqueiras et al., 2020).

Este interés por la alfabetización también se ha visto reflejado en algunos centros de salud y organizaciones de salud pública que han comenzado a usar Twitter para promover acciones de educación en salud, complementando medidas que se estaban realizando a través de otros canales y logrando impactar a un mayor número de personas (Xu et al., 2016). Además, frente a otras formas de difusión de información, una de las grandes ventajas de las redes sociales es la posibilidad de medición de la eficacia de las acciones, mediante, por ejemplo, la cuantificación de las interacciones de los usuarios con las distintas publicaciones ( $l i$ kes, retweets, compartir, comentar).

\subsection{La comunicación sobre la enfermedad del cáncer}

Dentro de los problemas de salud, el cáncer es una de las enfermedades que más circula en las redes sociales (de Oliveira, 2020) ya que es una enfermedad que afecta a más de 19 millones de personas en todo el mundo, siendo el cáncer de mama, el de pulmón y el de colon los tres más diagnosticados en el año 2020 según el National Institutes of Health (NIH, 2020). Es precisamente por su alta incidencia que se debe considerar un tema esencial desde el punto de vista de la salud pública, cuyo uno de sus objetivos principales es la promoción de la salud a través de acciones de información, educación y empoderamiento de las personas en temas de salud.

Según el Instituto Nacional del Cáncer (2020), es muy importante tratar el cáncer con una buena y correcta perspectiva comunicativa. Manifiestan las siguientes indicaciones en este sentido: la buena comunicación entre los pacientes de cáncer, familiares que los cuidan y el equipo de sanitarios; los pacientes oncológicos tienen necesidades especiales de comunicación; la comunicación es importante en los diferentes momentos de la atención del cáncer, es decir, cuando el paciente recibe su primer diagnóstico, cualquier momento en que se deben tomar decisiones acerca del tratamiento, después del tratamiento, cuando se habla sobre su eficacia, cualquier momento en que cambian las metas de la atención, cuando el paciente da a conocer sus deseos en cuanto a sus instrucciones por adelantado como por ejemplo, un testamento vital.

Por lo tanto, si la comunicación es esencial en todo el proceso (sobre todo cuando se deben tomar decisiones significativas), Twitter como red social puede contribuir a fomentar, dinamizar y agilizar la información, opiniones y en definitiva todo el proceso comunicativo entre los diferentes agentes implicados, ya que en investigaciones recientes se ha señalado su efectividad frente a otras redes sociales en la comunicación sobre cáncer (Vraga et al., 2018).

Diversos estudios ponen de manifiesto la abundancia de la información sobre esta enfermedad publicada en la red: pacientes que han sobrevivido a la enfermedad (Chou, 2011); información sobre el cáncer e internet (García-Mirón y Torres-Romay, 2020); sobre los procesos emocionales (Schmidt y Andrykowski, 2004); las relaciones y comunicación entre doctor y paciente de cáncer (Forguione-Pérez, 2015); beneficios y efectos adversos de tratamientos (Jiménez-León, 2015); cáncer de mama (Kern y Moro, 2012); cáncer y terapias en adolescentes (Haase, 2020); prevención (Plackett et al., 2020) y factores de riesgo (Vandertempel, 2015). 
El interés de este artículo radica en que todavía no se ha realizado ninguna revisión que trate de compilar los resultados de los estudios empíricos que analizan Twitter como medio de comunicación sobre cáncer. Por ese motivo, los objetivos específicos de este estudio son:

- Identificar en los artículos estudiados, el tipo de información (promoción/tratamientos) que se difunde a través de Twitter.

- Identificar las principales temáticas o enfoques estudiados en la literatura científica sobre el uso de Twitter en relación con el cáncer.

- Analizar las conclusiones de los artículos seleccionados sobre el uso de Twitter como herramienta de vigilancia de la salud.

- Examinar las conclusiones de los artículos estudiados para evaluar la eficacia del uso de Twitter como fuente de información en salud.

\section{Metodología}

Para esta investigación, se desarrolló una revisión bibliográfica de la literatura científica publicada durante un periodo de diez años (de 2009 a 2019) sobre temas de comunicación en salud a través de Twitter. La búsqueda se realizó en julio de 2020 en las bases de datos de Web of Science Core Collection y PubMed.

Para la realización de la búsqueda se acotó por la aparición en el título, en el abstract, en las palabras clave de autor y/o en las Keywords Plus de alguna de las palabras clave: "Health Communication" and "Twitter", por idiomas (inglés y español) y por tipo de artículo (Classical article). Estos términos fueron elegidos siguiendo la misma estructura descrita en Segado y Fernández (2015) combinando etiquetas globales como "Health Communication", y otras específicas que con el nombre concreto de la red Social "Twitter".

Se recuperaron un total de 107 artículos utilizando los términos de búsqueda establecidos inicialmente. Sus resúmenes se revisaron manualmente y se eliminaron artículos que no cumplían con los criterios de inclusión/exclusión establecidos previamente, es decir, artículos empíricos que se refieran específicamente a la comunicación en salud transmitida a través de Twitter. Esto dejó fuera de la muestra a 87 artículos que 1) mencionaron el concepto de comunicación en salud en Twitter de forma tangencial, sin ser objeto específico de estudio; 2) artículos que estaban restringidos a comunicación médica o especializada;3) artículos que se repetían en las distintas bases de datos.

Finalmente, se realizó un análisis de contenido de los 20 artículos centrados en la difusión de información sobre cáncer a través de Twitter. Para ello se incluyeron tanto los artículos que contuviesen en el título la palabra clave "cáncer", así como artículos centrados en factores de riesgo para el desarrollo de dicha enfermedad (ver Figura 1). 
Figura 1. Criterios de inclusión y exclusión aplicados en la revisión (2009/2019)

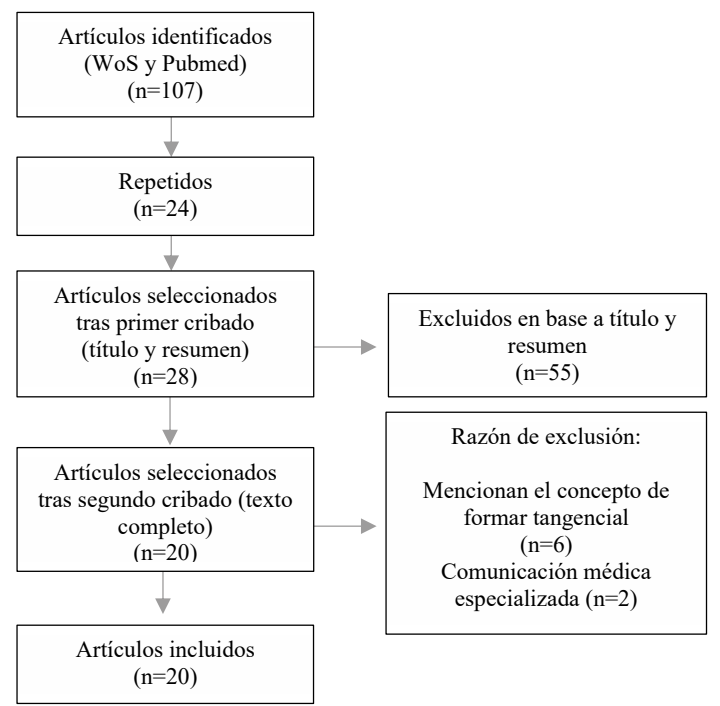

Fuente: elaboración propia

Para el análisis de contenido se elaboró un protocolo de recogida de información que contenía las siguientes variables:

1. Año de publicación del artículo.

2. Número de investigadores.

3. Sexo del investigador principal.

4. Redes sociales estudiadas.

5. Tópico que se estudia.

6. Metodología de análisis del artículo.

7. Tamaño muestral del artículo.

8. País/países que constituyen en universo de estudio del artículo.

9. Público al que se dirigen las acciones de comunicación.

10. Enfoque del artículo: Información/Educación/Empoderamiento.

11. Información farmacológica (nombres comerciales, efectos secundarios, eficacia y efectividad).

Por último, se clasificaron los artículos en función del enfoque (información, educación y empoderamiento) y la valencia de sus conclusiones (positiva o negativa) siguiendo las definiciones establecidas en la Tabla 1. 
Tabla 1. Enfoque y valencia de las conclusiones de los artículos incluidos en la revisión

\begin{tabular}{|l|l|l|}
\hline Uso de Twitter & $\begin{array}{l}\text { Valencia positiva } \\
\text { Descripción }\end{array}$ & $\begin{array}{l}\text { Valencia negativa } \\
\text { Descripción }\end{array}$ \\
\hline Información & $\begin{array}{l}\text { Constituye una fuente de información fiable sobre salud } \\
\text { y contribuye eficazmente a la promoción de conductas } \\
\text { saludables a través de la información. }\end{array}$ & $\begin{array}{l}\text { Necesita de mayor intervención de profesionales de salud } \\
\text { para mejorar la calidad de la información que se difunde } \\
\text { y promocionar conductas saludables. }\end{array}$ \\
\hline Educación & $\begin{array}{l}\text { Permite sensibilizar o visibilizar problemas de salud, } \\
\text { así como evaluar el impacto de las acciones de } \\
\text { sensibilización. }\end{array}$ & $\begin{array}{l}\text { Continúa infrarrepresentado o invisibilizando ciertos } \\
\text { problemas de salud o prevalencias. }\end{array}$ \\
\hline Empoderamiento & $\begin{array}{l}\text { Fomenta la comunicación, las redes de apoyo y el debate } \\
\text { entre diferentes actores y permite la comunicación en } \\
\text { situaciones de emergencia. }\end{array}$ & $\begin{array}{l}\text { Comunicación unidireccional que no fomenta el diálogo } \\
\text { ni contribuye a mejorar la comunicación entre los } \\
\text { diferentes actores o en situaciones sanitarias críticas. }\end{array}$ \\
\hline
\end{tabular}

Fuente: elaboración propia

Para evitar la variación inter-observador-a en la codificación de la información se realizó un análisis de concordancia sobre una muestra del $20 \%$ del total del universo estudiado, obteniendo un 81,3\% de acuerdo (BG y BC).

La información se registrará en una base de datos de SPSS, versión 15, que posteriormente se utilizó para el análisis de los resultados.

\section{Resultados}

Durante el periodo comprendido entre 2009 y 2019 encontramos un total de 20 artículos que analizaban la comunicación sobre cáncer en Twitter (Tabla 2).

Tabla 2. Artículos revisados centrados en la difusión de información sobre cáncer a través de Twitter (2009/2019)

\begin{tabular}{|l|l|}
\hline APELLIDO, AÑ & TÍTULO DEL ARTÍCULO \\
\hline VILLA, 2012 & $\begin{array}{l}\text { Redes sociales de internet en difusión antitabáquica: la experiencia de la Clínica Contra el Tabaquismo del Hospital } \\
\text { General de México «Eduardo Liceaga» }\end{array}$ \\
\hline MAHONEY, 2015 & $\begin{array}{l}\text { The Digital Distribution of Public Health News Surrounding the Human Papillomavirus Vaccination:A Longitudinal } \\
\text { Infodemiology Study }\end{array}$ \\
\hline BRAVO, 2015 & $\begin{array}{l}\text { Tweeting About Prostate and Testicular Cancers: What Are Individuals Saying in Their Discussions About the 2013 } \\
\text { Movember Canada Campaign? }\end{array}$ \\
\hline $\begin{array}{l}\text { VANDERTEMPEL, } \\
2015\end{array}$ & Vape, quit, tweet? Electronic cigarettes and smoking cessation on Twitter \\
\hline
\end{tabular}

$1 \quad$ Apellido del primer autor y año de publicación del artículo revisado. 


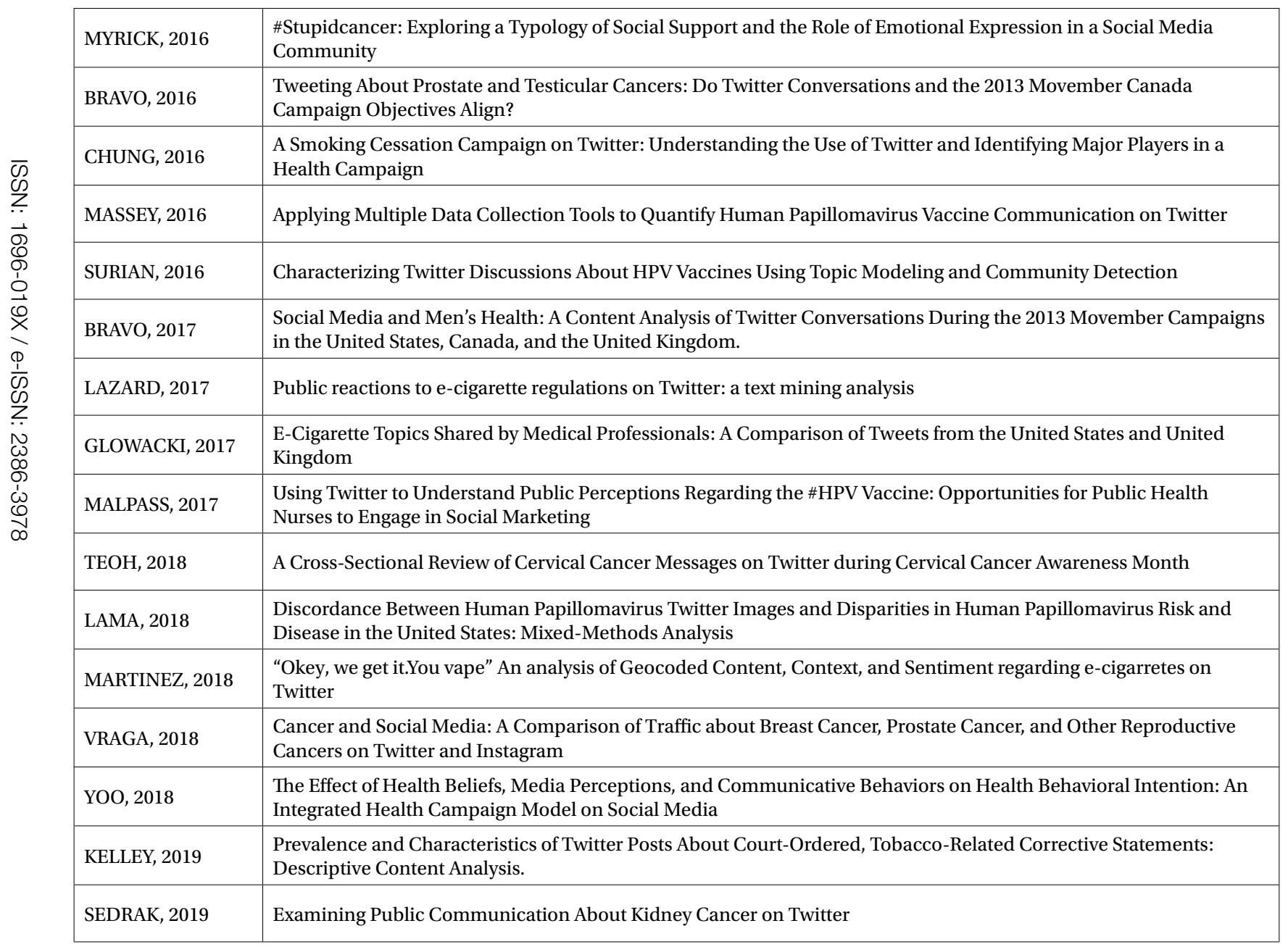

\section{Fuente: elaboración propia}

El 70\% de los artículos revisados fueron publicados en el periodo que va de 2016 a 2018, mientras que el inicio del periodo de análisis más frecuentemente estudiado fue entre los años 2013 al 2015 (20\% cada año). El 60\% de los artículos seleccionados estaban centrados en EE.UU., el 10\% en Canadá, el 5\% en México, el 5\% en Suiza y un 5\% no contenía esta información. Por último, un 15\% de los artículos no se especificaba un país en concreto, sino que estaban centrados en países de habla inglesa (ver Tabla 3). 
Tabla 3. Frecuencia y porcentaje de artículos revisados por temática y país de estudio (2009/2019)

\begin{tabular}{|c|c|c|c|c|c|c|c|c|}
\hline $\begin{array}{l}\text { País de estudio } \\
\text { del artículo }\end{array}$ & $\begin{array}{c}\text { Cigarrillos } \\
\text { electrónicos } \\
n(\%)\end{array}$ & $\begin{array}{c}\text { Vacuna } \\
\text { contra el } \\
\text { VPH } \\
n(\%)\end{array}$ & $\begin{array}{c}\text { Campañas } \\
\text { anti- } \\
\text { tabáquicas } \\
\mathrm{n}(\%)\end{array}$ & $\begin{array}{c}\text { Riesgo } \\
\text { consumo } \\
\text { tabaco } \\
\text { n }(\%)\end{array}$ & $\begin{array}{c}\text { Cáncer } \\
\text { reproductivo } \\
\mathrm{n}(\%)\end{array}$ & $\begin{array}{c}\text { Cáncer de } \\
\text { riñón } \\
\mathrm{n}(\%)\end{array}$ & $\begin{array}{c}\text { Cáncer } \\
\text { n (\%) }\end{array}$ & $\begin{array}{l}\text { Total } \\
\mathrm{n}(\%)\end{array}$ \\
\hline EE. UU. & $3(75)$ & $4(57,1)$ & $1(50)$ & $1(100)$ & $1(25)$ & $1(100)$ & $1(100)$ & $12(60)$ \\
\hline Canadá & 0 & 0 & 0 & 0 & $2(50)$ & 0 & 0 & $2(10)$ \\
\hline México & 0 & 0 & $1(50)$ & 0 & 0 & 0 & 0 & $1(5)$ \\
\hline Suiza & $1(25)$ & 0 & 0 & 0 & 0 & 0 & 0 & $1(5)$ \\
\hline $\begin{array}{l}\text { Países de habla } \\
\text { inglesa }\end{array}$ & 0 & $2(28,6)$ & 0 & 0 & $1(25)$ & 0 & 0 & $3(15)$ \\
\hline No identificado & 0 & $1(14,3)$ & 0 & 0 & 0 & 0 & 0 & $1(5)$ \\
\hline Total & $4(20)$ & $7(35)$ & $2(10)$ & $1(5)$ & $4(20)$ & $1(5)$ & $1(5)$ & $20(100)$ \\
\hline
\end{tabular}

Fuente: elaboración propia

El 45\% de los artículos tenían cuatro (25\%) o 6 firmantes (20\%) y en el 80\% del total de la muestra la investigadora principal es una mujer. La temática en la que mayor número de hombres aparecen como primeros firmantes es la vacuna contra el VPH (42,9\%) y los cigarrillos electrónicos (25\%), mientras que en el resto de las temáticas las mujeres aparecen como primeras firmantes en el $100 \%$ de los casos.

La gran mayoría de los artículos incluidos en la revisión estudia únicamente Twitter (85\%) y el 15\% restante combina el estudio de Twitter con el de otra red social como Instagram (5\%), Facebook (5\%) y Google News (5\%).

En el $70 \%$ de los artículos el tema central del análisis fueron los factores de riesgo asociados al cáncer, siendo la vacuna contra el VPH el tema más estudiado en los 20 artículos analizados (35,7\%), seguido de los cigarrillos electrónicos (28,6\%). Por otro lado, cuando el artículo se centra en la propia enfermedad, los cánceres reproductivos (mama, próstata, testículos) son los que aparecen con mayor frecuencia, suponiendo el 66,7\% del total de los 6 artículos centrados en el cáncer como temática (ver Tabla 4).

Tabla 4. Artículos centrados en la difusión de información sobre cáncer en Twitter

\begin{tabular}{|c|c|c|c|}
\hline & Temática & Tópico específico & $\mathbf{N}(\%)$ \\
\hline \multirow{4}{*}{$\begin{array}{l}\text { Factores de riesgo para el } \\
\text { desarrollo de cáncer }\end{array}$} & \multirow{3}{*}{ Tabaco } & Cigarrillos Electrónicos & $4(20)$ \\
\hline & & Campañas antitabáquicas & $2(10)$ \\
\hline & & Riesgos del consumo de tabaco & $1(5)$ \\
\hline & VPH & Vacuna contra el VPH & $7(35)$ \\
\hline \multirow{3}{*}{ Enfermedad } & \multirow{3}{*}{ Cáncer } & Cáncer reproductivo (mama, próstata, testículos, cuello de útero) & $4(20)$ \\
\hline & & Cáncer de riñón & $1(5)$ \\
\hline & & Sin especificar tipo & $1(5)$ \\
\hline
\end{tabular}

Fuente: elaboración propia 
No es frecuente la aparición de nombres comerciales de medicamentos, que solo se menciona en un artículo sobre la vacuna contra el VPH. Sin embargo, en un $25 \%$ del total de artículos revisados se mencionan efectos adversos de productos o medicamentos para la prevención del cáncer, en un $35 \%$ se menciona su eficacia y en un $5 \%$ la efectividad. Al diferenciar por tópico específico, vemos como esta información aparece fundamentalmente en relación a la vacuna contra el VPH, donde un $57,1 \%$ de los artículos mencionan los efectos adversos y un 85,7\% mencionan la eficacia. También en el caso de los cigarrillos electrónicos en un 25\% de los artículos revisados centrados en este tema, se mencionan tanto los efectos adversos como la eficacia (ver Tabla 5).

Tabla 5. Información sobre productos o tratamientos farmacológicos para la prevención del cáncer

\begin{tabular}{|c|c|c|c|c|c|c|c|c|}
\hline & \multicolumn{2}{|c|}{ Nombres comerciales } & \multicolumn{2}{|c|}{ Efectos adversos } & \multicolumn{2}{|c|}{ Eficacia } & \multicolumn{2}{|c|}{ Efectividad } \\
\hline & $\begin{array}{c}\mathrm{Si} \\
\mathrm{n}(\%)\end{array}$ & $\begin{array}{c}\text { No } \\
\text { n (\%) }\end{array}$ & $\begin{array}{c}\mathrm{Si} \\
\mathrm{n}(\%)\end{array}$ & $\begin{array}{c}\text { No } \\
\text { n (\%) }\end{array}$ & $\begin{array}{c}\mathrm{Si} \\
\mathrm{n}(\%)\end{array}$ & $\begin{array}{c}\text { No } \\
\text { n (\%) }\end{array}$ & $\begin{array}{c}\mathrm{Si} \\
\mathrm{n}(\%)\end{array}$ & $\begin{array}{c}\text { No } \\
\text { n (\%) }\end{array}$ \\
\hline Cigarrillos electrónicos & 0 & $4(100)$ & $1(25)$ & $3(75)$ & $1(25)$ & $3(75)$ & 0 & $4(100)$ \\
\hline Campaña antitabáquica & 0 & $2(100)$ & 0 & $2(100)$ & 0 & $2(100)$ & 0 & $2(100)$ \\
\hline Riesgos de tabaco & 0 & $1(100)$ & 0 & $1(100)$ & 0 & $1(100)$ & 0 & $1(100)$ \\
\hline Vacuna contra el VPH & $1(14,3)$ & $6(85,7)$ & $4(57,1)$ & $3(42,9)$ & $6(85,7)$ & $1(14,3)$ & $1(14,3)$ & $6(85,7)$ \\
\hline Cáncer reproductivo & 0 & $4(100)$ & 0 & $4(100)$ & 0 & $4(100)$ & 0 & $4(100)$ \\
\hline Cáncer de riñón & 0 & $1(100)$ & 0 & $1(100)$ & 0 & $1(100)$ & 0 & $1(100)$ \\
\hline Cáncer & 0 & $1(100)$ & 0 & $1(100)$ & 0 & $1(100)$ & 0 & $1(100)$ \\
\hline
\end{tabular}

Fuente: elaboración propia

En lo relativo a las conclusiones de los artículos, se han encontrado un total de 26 enfoques, siendo la clasificación de enfoques no excluyentes. De ellos, un 64,3\% concluyen afirmando que Twitter es una herramienta efectiva para educar en salud. Sin embargo, también un 58,3\% de los artículos concluyen afirmando que la calidad de la información es insuficiente y es necesaria una mayor intervención por parte de los profesionales de la salud para mejorar la calidad de la información que se difunde. Respecto a los 9 artículos que se enfocan en el empoderamiento, encontramos que las opiniones están bastante polarizadas siendo un $44,4 \%$ de los artículos los que concluyen afirmando que contribuye al empoderamiento de los usuarios y un 55,6\% de los artículos los que concluyen afirmando lo contrario (ver Tabla 6).

Tabla 6. Usos y valencias de Twitter en Health Communication identificados en las conclusiones de los artículos revisados

\begin{tabular}{|c|c|c|c|c|c|c|}
\hline \multirow[t]{2}{*}{ Uso de Twitter } & \multicolumn{2}{|c|}{ Valencia positiva } & \multicolumn{2}{|c|}{ Valencia negativa } & \multicolumn{2}{|c|}{ Total } \\
\hline & No & $\%$ & No & $\%$ & No & $(\%)$ \\
\hline Información & 1 & 7,1 & 7 & 58,3 & 8 & 30,8 \\
\hline Educación & 9 & 64,3 & 0 & 0 & 9 & 34,6 \\
\hline Empoderamiento & 4 & 28,6 & 5 & 41,7 & 9 & 34,6 \\
\hline Total & 14 & 100 & 12 & 100 & 26 & 100 \\
\hline
\end{tabular}

Fuente: elaboración propia 


\section{Discusión}

De acuerdo con los resultados obtenidos podemos afirmar que en las bases de datos consultadas se han encontrado un escaso número de artículos empíricos centrados en el análisis de la comunicación sobre cáncer a través de Twitter. Este resultado coincide con revisiones bibliográficas previas sobre el análisis de la comunicación en salud a través de esta red social digital, donde comparativamente se observa que el cáncer ha tenido menor presencia que otros problemas de salud como las enfermedades infecciosas, psiquiátricas o neurológicas, que han sido foco de estudio con mayor frecuencia (Sinnenberg et al., 2017).

Por otro lado, también conviene destacar que la mayoría de los análisis efectuados en los artículos que componen nuestro universo de estudio han puesto el foco en países de habla inglesa o en análisis de la comunicación en inglés, teniendo poca representación el análisis de la comunicación en otros países o con otros idiomas, como por ejemplo el español, lo que evidencia un sesgo idiomático en la investigación sobre comunicación y salud, sesgo ya identificado anteriormente en el área de comunicación (De la Torre-Espinosa et al., 2019).

Además, también se observa como el estudio de la difusión de información sobre cáncer en Twitter es una temática de trabajo ampliamente feminizada, ya que son mayoritariamente las mujeres las que lideran las investigaciones sobre este tema, independientemente del enfoque o tipo de cáncer en el que se centre el análisis del artículo. Estos resultados pueden corresponderse también con el mayor número de profesionales sanitarias mujeres (Witter et al., 2017), lo que puede implicar también un mayor número de investigadoras en esta área. No obstante, a pesar de las diferencias por sexo entre los primeros firmantes de los artículos revisados, se han estudiado por igual problemas de salud que repercuten a hombres y a mujeres, ya que tanto la comunicación sobre el cáncer de mama como la comunicación sobre el cáncer de próstata han sido dos de los tipos de cánceres más estudiados.

La mayoría de los artículos se han centrado en analizar la comunicación en relación con factores de riesgo como el tabaco o el VPH, no siendo tan frecuente centrarse en el cáncer como enfermedad en sí misma. Por lo tanto, cuando se analiza la comunicación en salud a través de Twitter, el foco de atención es más la prevención que su eficacia para la creación de redes de apoyo o la valoración de su uso como herramienta para la búsqueda de información sobre la enfermedad.

En este sentido se debe señalar que a pesar de que pocos artículos destacan la presencia de nombres comerciales de tratamientos farmacológicos, si que es frecuente la aparición de argumentos centrados en los efectos adversos que pueden generar productos como las vacunas o los cigarrillos electrónicos. Esto puede estar indicando que muchas de las investigaciones realizadas han estudiado el uso de Twitter como herramienta de vigilancia de la salud, mediante el seguimiento de la comunicación para la identificación de necesidades sobre temas de salud que puedan ser sensibles o controvertidos o sobre los que exista polémica.

En el caso de la selección temática, hay que destacar que el alto número de artículos que se centran en el consumo de tabaco como factor de riesgo para el desarrollo de un cáncer respiratorio (bronquios, tráquea o pulmón) se ve justificado por su alta incidencia y mortalidad a nivel mundial según la World Health Organization (WHO, 2020), al igual que sucede en el caso del cáncer de mama y próstata, ambos con una alta incidencia. Sin embargo, este mismo criterio no podría aplicarse en el caso del VPH, factor de riesgo para el desarrollo de cáncer de cuello uterino, ya que este tipo de cáncer no se encuentra ni entre los más diagnosticados ni entre los que tienen una mayor mortalidad (American Cancer Society, 2020). 
Respecto a las conclusiones de los artículos, muchos autores destacan que Twitter es una herramienta valiosa para educar en temas de salud, concretamente sobre la enfermedad del cáncer, permitiendo sensibilizar o visibilizar los factores de riesgo que repercuten en el desarrollo de la enfermedad y evaluar el impacto de dichas acciones. Sin embargo, también se destaca que la información sobre temas de salud a menudo es incorrecta y que es necesaria una mayor intervención por parte de los profesionales sanitarios. Se pone así de manifiesto que las redes sociales son una herramienta de apoyo que debe ir de la mano de otras acciones, es decir, es un refuerzo, pero no debe ser considerada una única medida sino más bien es una herramienta a través de la cual se puede medir el impacto de acciones de comunicación que se han realizado por otros medios.

Además, según los resultados obtenidos, tampoco existe consenso sobre la utilidad de Twitter para empoderar a la ciudadanía en temas de salud, ya que a pesar de que una de las principales ventajas de las redes sociales es la bidireccionalidad de la comunicación, también muchos artículos concluyen afirmando que esta sigue siendo mayoritariamente unidireccional y la mayoría de los usuarios se limitan a volcar opiniones y experiencias personales, no evitando, en este caso, la soledad del enfermo oncológico, que necesita para que la comunicación sea efectiva, entre otras cosas, la preservación de la esperanza y el reconocimiento emocional (Cunill y Serdà, 2011). No obstante, para el verdadero empoderamiento también es necesario reforzar la alfabetización mediática que permita evaluar la información de manera crítica, evitando así la confusión al exponerse a opiniones que pueden estar sesgadas o fundamentados en experiencias aisladas.

Una de las principales limitaciones de esta revisión bibliográfica es el reducido tamaño de la muestra que impide generalizar acerca de la calidad de la información sobre cáncer en Twitter. No obstante, es este trabajo abre una primera vía de investigación que permite compilar los análisis realizados hasta el momento y extraer unas primeras conclusiones al respecto. Además, hay que tener en cuenta que solo se han analizado aquellos estudios que se centraban en Twitter por lo que los resultados no se pueden extrapolar a otras redes sociales con mayor número de usuarios como Facebook o Instagram.

\section{Conclusiones}

La mayoría de los artículos revisados relacionados con la comunicación sobre cáncer a través de Twitter se han centrado en el análisis de la comunicación relativa a factores de riesgo para el desarrollo de la enfermedad, fundamentalmente tabaco y VPH, más que en el cáncer como enfermedad en sí misma, por lo que esto puede estar indicando que, desde el ámbito académico, el foco de atención ha estado centrado en temáticas preventivas.

Se señala la necesidad de una mayor alfabetización mediática que permita a los pacientes oncológicos evaluar la información de manera crítica, al exponerse a lo que pueden ser opiniones parciales o sesgadas. A su vez, también hay que destacar la eficacia de su uso como herramienta de apoyo, no solo para la difusión de acciones de sensibilización o visibilización sino también para la medición del impacto de acciones de comunicación realizadas por otros medios. Por último, en lo relativo a su eficacia como herramienta de empoderamiento en la creación de redes de apoyo que eviten la soledad del enfermo, no existe consenso en los artículos analizados, ya que algunos autores señalan que frecuentemente se produce unidireccionalidad en la comunicación.

Como futuras líneas de investigación consideramos que sería interesante realizar una revisión bibliográfica sobre las temáticas de salud más abordadas en otras redes sociales con el objetivo de poder hacer una comparativa entre ellas. Así mismo, también 
se podría ampliar la revisión bibliográfica a otros problemas de salud para determinar si existe correspondencia entre el peso de las enfermedades con mayor incidencia y/o prevalencia y su presencia en los debates sobre salud que se generan en Twitter.

\section{Agradecimientos}

Este artículo ha sido traducido por Sophie Phillips.

Este artículo ha sido financiado con cargo al grupo de investigación Comunicación y Salud en Redes Sociales (COYSADI), desarrollado por el grupo de investigación Comunicación y Sociedad Digital(COYSODY) en el marco de la convocatoria Proyectos I+D+i Retos 2020-2021 de la Universidad Internacional de la Rioja (UNIR).

\section{Referencias bibliográficas}

American Cancer Society (20 de diciembre de 2020). Cancer Facts and Figures 2020. Atlanta, Ga: American Cancer Society. https:// www.cancer.org/cancer.html

Calvo, E. y Aruguete, N. (2020). Fake news, trolls y otros encantos: cómo funcionan. Siglo XXI Editores.

Castells, M. (2012). Redes de indignación y esperanza: Los movimientos sociales en la era de internet. Alianza Editorial.

Chou, WS., Hunt, Y., Folkers, A. y Augustson, E. (2011). Cancer Survivorship in the Age of YouTube and Social Media: A Narrative Analysis. Journal of Medical Internet Research, 13(1): e7. https://doi.org/10.2196/jmir.1569

Crua, C. (2020). (03 de mayo de 2021). ¿Cómo son los usuarios de Twitter y cómo se comportan con las marcas?. https://www. iebschool.com/blog/estadisticas-usuarios-twitter-como-son-redes-sociales/

Cunill, M., y Serdà, B. C. (2011). Características de la comunicación con enfermos de cáncer en el contexto sanitario y familiar. Psicooncología, 8(1), 65. https://doi.org/10.5209/rev_PSIC.2011.v8.n1.6

Curioso, W.H. y Carnero, A. M. (2011). (24 de mayo de 2021). Promoviendo la investigación en salud con Twiter. http://www. scielo.org.pe/scielo.php?pid=S1018-130X2011000300006\&script=sci_arttext

De la Torre-Espinosa, M., Repiso, R., y Díaz, J. M. (2019). Factor de Impacto y comportamiento bibliométrico de las revistas de “Film, Radio \& Television" de Web of Science. Revista Española de Documentación Científica, 42(3), 243. https://doi.org/10.3989/ redc.2019.3.1630

De Oliveira, MD., de Souza, MP., Fuentes, JA. y Pessalacia, J. (2020). (26 de mayo de 2021). Experiências de pacientes com câncer divulgadas nas redes sociais virtuais. Revista Cubana de Información en Ciencias de la Salud, 31(3), e1580. Epub 30 de octubre de 2020. http://scielo.sld.cu/scielo.php?script=sci_arttext\&pid=S2307-21132020000300013\&lng=es\&tlng=pt.

Fernández, C., Jiménez, A. y Granados, M. (2014). IV Jornada Nacional de Ciencias de la Información en Salud. Salud 2.0: un desafío de comunicación con el paciente.

Forguione-Pérez VP. (2015). Comunicación entre médico y paciente: más allá de una consulta, un proceso educativo. MÉD. UIS.,28(1),7-13.

Gage-Bouchard, E. A., LaValley, S., Warunek, M., Beaupin, L. K. y Mollica, M. (2018). Is cancer information exchanged on social media scientifically accurate?. Journal of Cancer Education, 33(6), 1328-1332. https://doi .org/10.1007/s13187-017-1254-z 
Galeano, S. (2021). (04 de mayo de 2021). El número de usuarios de internet en el mundo crece un 7,3\% y alcanza los 4.660 millones. https://marketing4ecommerce.net/usuarios-de-internet-mundo/

García, L. (2018). (05 de mayo de 2021). Salud sin bulos: una iniciativa para erradicar rumores falsos en la red. https://www. agenciasinc.es/Noticias/Salud-sin-bulos-una-iniciativa-para-erradicar-rumores-falsos-en-la-red

García-Mirón, S. y Torres-Romay, E. (2020). Información sobre cáncer en Internet. Revista de Comunicación y Salud, 10(3), 1-22. file://C:/Users/Admin/Downloads/Dialnet-InformacionSobreCancerEnInternet-7711694.pdf

González, P. (2019). (07 de mayo de 2021). El cáncer se mueve en redes. https://www.efesalud.com/cancer-redes-sociales/

Haase, et al. (2020). Adolescent/Young Adult Perspectives of a Therapeutic Music Video Intervention to Improve Resilience During Hematopoietic Stem Cell Transplant for Cancer. Journal of Music Therapy, 57(1), 3-33. https://doi.org/10.1093/jmt/thz014

Haase, J. E., Robb, S. L., Burns, D. S., Stegenga, K., Cherven, B., Hendricks-Ferguson, Roll, L, Docherty, S y Phillips, C. (2020). Adolescent/young adult perspectives of a therapeutic music video intervention to improve resilience during hematopoietic stem cell transplant for cancer. Journal of music therapy, 57(1), 3-33. https://doi.org/10.1093/jmt/thz014

Instituto Nacional del Cáncer (2020). (08 de mayo de 2021). Comunicación en la atención del cáncer. Versión para pacientes. https://www.cancer.gov/espanol/cancer/sobrellevar/adaptacion-al-cancer/comunicacion-pdqJiménez

Jiménez-León, M. (2015). (02 de mayo de 2021). Beneficios y efectos adversos del tratamiento con opioides en el dolor por cáncer. https://hdl.handle.net/10953.1/1654

Keckley, PH. y Hoffmann, M. (2010). (25 de mayo de 2021). Social Networks in Health Care: Communication, collaboration and insights. http://www.healthcarevisions.snapmonkey.net/f/2010_Deliotte_Social_Networks.pdf

Kern, E. y Moro, L. (2012). Factores psicosociales relacionados con el autocuidado en la prevención, tratamiento y postratamiento del cáncer de mama. Psicooncología, 9(2-3), 453-465. https://doi.org/10.5209/rev_PSIC.2013.v9.n2-3.40916

Martín, A. G., y Tyner, K. (2012). Media education, media literacy and digital competence. Comunicar. Media Education Research Journal, 20(1).

Matarín, T. (2015). Redes sociales en prevención y promoción de la salud. Una revisión de la actualidad. Revista Española de Comunicación en salud, 6(1), 62-69.

National Institutes of Health (NIH) (2020). (10 de abril de 2021). Naturaleza del cáncer. https://www.cancer.gov/espanol/cancer/ naturaleza/estadisticas

Paakkari, L., y Okan, O. (2020). COVID-19: health literacy is an underestimated problem. The Lancet Public Health, 5(5), e249-e250. https://doi.org/10.1016/S2468-2667(20)30086-4

Piqueiras, P., de Marchis, G., y Cuesta, V. (2020). Análisis del contenido publicado en YouTube, Facebook e internet sobre vacunas y anti vacunas. Revista de Comunicación y Salud, 10(1), 67-90. https://doi.org/10.35669/rcys.2020.10(1).67-90

Plackett, R., Kasuhal, A., Kasianos, A., Cross, A, lewins, D., Sheringham, J. (2020). Use of Social Media to promote cancer screening and early diagnosis: scoping review. Journal of Medical Internet Research, $22(11)$ : e21582. https://doi.org/10.2196/21582 
Rufai, S. R., y Bunce, C. (2020). World leaders' usage of Twitter in response to the COVID-19 pandemic: a content analysis. Journal of Public Health, 42(3), 510-516.

Sanz, E. y Delgado, D. (04 de mayo de 2021). ¿Para qué se utiliza Twitter?. https://www.muyinteresante.es/tecnologia/articulo/ ipara-que-se-utiliza-twitter

Sanders, K., Valle, M. S., Viñarás, M., \& Llorente, C. (2015). Do we trust and are we empowered by “Dr. Google”? Older Spaniards' uses and views of digital healthcare communication. Public Relations Review, 41(5), 794-800.

Schapira, L. (2019). (02 de mayo de 2021). Seis maneras en las que los familiares y amigos pueden ayudar cuando usted tiene cáncer. https://www.cancer.net/es/blog/2019-02/6-maneras-en-las-que-los-familiares-y-amigos-pueden-ayudar-cuando-usted-tiene-c\%C3\%Alncer

Schmidt, J. E. y Andrykowski, M. A. (2004). The Role of Social and Dispositional Variables Associated With Emotional Processing in Adjustment to Breast Cancer: An Internet-Based Study. Health Psychology, 23(3), 259-266.

https://doi.org/10.1037/0278-6133.23.3.259

Segado, F. y Fernández, E. (2015). Social media and television: a bibliographic review based on the Web of Science. Profesional de la Información, 24(3), 235-245.

Sinnenberg, L., Buttenheim, AM., Padrez, K., Mancheno, C., Ungar, L., y Merchant, R. M. (2017). Twitter as a tool for health research: a systematic review. American Journal of Public Health, 107(1), e1-e8. https://doi.org/10.2105/AJPH.2016.303512

Sued, G. E., y Cebral, M. (2020). Voces autorizadas en Twitter durante la pandemia de COVID-19: actores, léxico y sentimientos como marco interpretativo para usuarios ordinarios. Revista de Comunicación y Salud, 10(2), 549-568. https://doi.org/10.35669/ rcys.2020.10(2).549-568

Vandertempel, (2015). Vape, quit, tweet? Electronic cigarettes and smoking cessation on Twitter. International Journal of Public Health, 61(2). https://doi.org/10.1007/s00038-016-0791-2

Vraga, E. K., Stefanidis, A., Lamprianidis, G., Croitoru, A., Crooks, A. T., Delamater, P. L., PFOSER, D., Radzikowski J.R., Jacobsen K.H. (2018). Cancer and social media: A comparison of traffic about breast cancer, prostate cancer, and other reproductive cancers on Twitter and Instagram. Journal of Health Communication, 23(2), 181-189.

We Are Social (15 de abril de 2021). Digital in 2021: Global Overview Report. https://datareportal.com/reports/ digital-2021-global-overview-report

Witter, S., Namakula, J., Wurie, H., Chirwa, Y., So, S., Vong, S. Ros, B., Buzuzi, S. y Theobald, S. (2017). The gendered health workforce: mixed methods analysis from four fragile and post-conflict contexts. Health Policy and Planning, 32(5), 52-62. https://doi. org/10.1093/heapol/czx102

World Health Organization (WHO) (2020). (01 de abril de 2021). The top 10 causes of death. https://www.who.int/news-room/ fact-sheets/detail/the-top-10-causes-of-death

Xu, S., Markson, C., Costello, K. L., Xing, C. Y., Demissie, K., y Llanos, A. A. (2016). Leveraging social media to promote public health knowledge: example of cancer awareness via Twitter. JMIR Public Health and Surveillance, 2(1). https://doi.org/10.2196/ publichealth.5205 
